
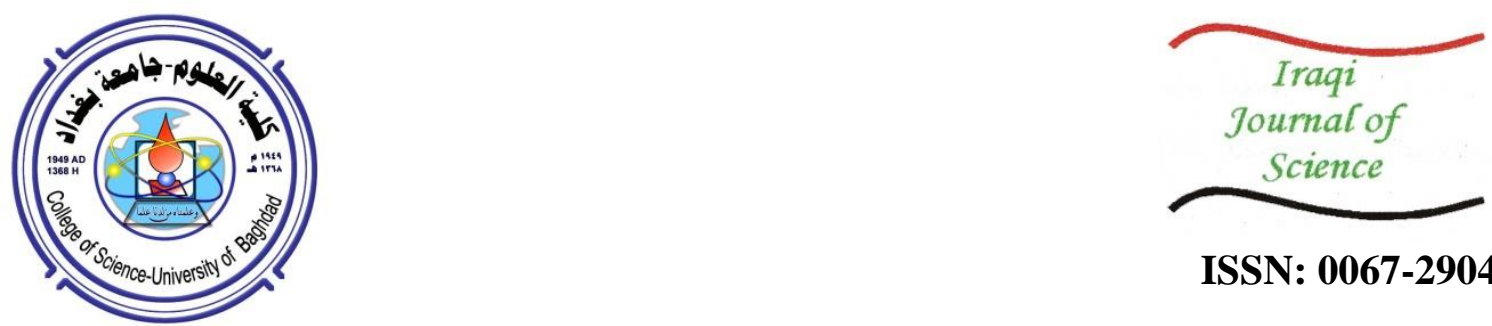

ISSN: 0067-2904

\title{
Placement Inverse Source Problem under Partition Hyperbolic Equation
}

\author{
Shilan Othman Hussein \\ Department of Mathematics, College of Science, University of Sulaymaniyah, Kurdistan Region, Iraq
}

Received: 2/9/2020

Accepted: 30/11/2020

\begin{abstract}
In this article, the inverse source problem is determined by the partition hyperbolic equation under the left end flux tension of the string, where the extra measurement is considered. The approximate solution is obtained in the form of splitting and applying the finite difference method (FDM). Moreover, this problem is ill-posed, dealing with instability of force after adding noise to the additional condition. To stabilize the solution, the regularization matrix is considered. Consequently, it is proved by error estimates between the regularized solution and the exact solution. The numerical results show that the method is efficient and stable.
\end{abstract}

Keywords: Inverse force hyperbolic problem; Regularization method; the left end flux tension of the string.
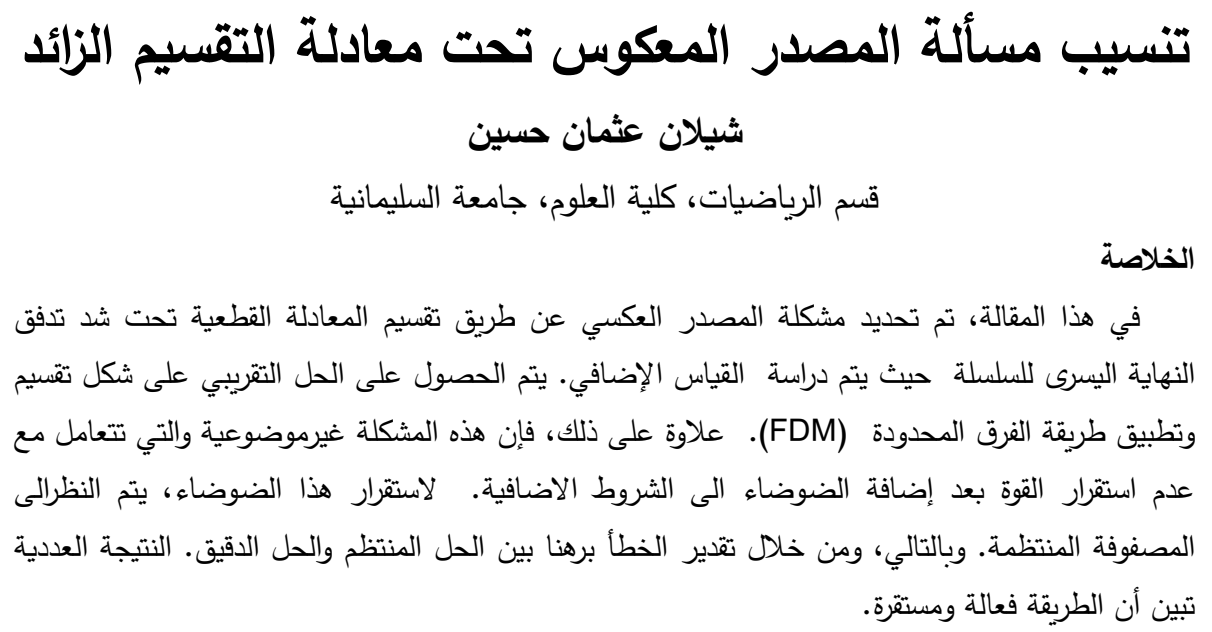

\section{Introduction}

The inverse force problem is described by partial differential equations and has been noticed recently because of its wide use in science, medicine, and mathematics fields. Many papers were dedicated to the optimal solution for source problems with different boundary conditions and numerical methods. In $[1,2]$, the boundary element method (BEM) was combined with a separation variable, where the left end Dirichlet or Neumann conditions, as additional measurements, were considered. Sequentially, in [3], the BEM was switched to the finite element method (FEM). Meanwhile, the papers $[4,12]$ were based on the right end Dirichlet or Neumann boundary conditions for extra conditions, whereas [5] focused on unusual boundary conditions with some theorems of existence and uniqueness, which were also provided in [5-8]. In addition, this approach was also used in physics $[9,10,12]$.

In this research, the existing results mainly focus on the finite difference method (FDM) for both partitions, which comes from splitting the wave equation. Moreover, the zeroth order of regularization

*Emails: shilan.husen@univsul.edu.iq 
is studied when the amount of noise is added to the extra boundary condition. This paper is organized as follows. We introduce the mathematical formulation in Section 2. In Section 3, numerical results are introduced to show that the method is stable. The conclusions are presented in Section 4.

\section{Mathematical Formulations}

In this article, it is required to find $f(x)$ subject to

$$
\begin{gathered}
u_{t t}(x, t)=\nabla^{2} u(x, t)+f(x) h(t), \quad(x, t) \in Z, \\
u(x, 0)=u_{0}(x), \quad u_{t}(x, 0)=v_{0}(x), \quad x \in Q, \\
u(0, t)=p_{0}(t), \quad u(L, t)=p_{L}(t), \quad t \in[0, T],
\end{gathered}
$$

where $(u(x, t)$ and $f(x) h(t))$ are the displacement and force, respectively, defining the points $(x, t) \in Z, x \in Q$, and $t \in(0, T) . Q \subset R^{n}$ is a bounded domain with a smooth boundary $\partial Q ; Z=Q \times$ $(0, T)$ which is a cylinder. $u_{0}$ and $v_{0}$ represent the initial displacement and velocity, respectively. Furthermore, for solving the inverse problem above, we need to have the following extra measurements in the role of the flux tension of the string at the left end $[1-4,11]$ :

$$
u_{x}(0, t)=q_{0}(t), \quad t \in(0, T) \text {. }
$$

We introduce the approach of solving (1)-(4) by partitioning $u(x, t)$ to $v(x, t)$ and $w(x, t)$ (i.e. $u=v+w)$ [6]. Consequently, by organizing (1)-(4) as in [1-4,6,11], we have:

$$
\begin{aligned}
& \left\{\begin{array}{l}
v_{t t}(x, t)=\nabla^{2} v(x, t), \\
w_{t t}(x, t)=\nabla^{2} w(x, t)+f(x) h(t),
\end{array}\right. \\
& v(x, 0)=u_{0}(x), \quad u_{t}(x, 0)=v_{0}(x), \quad x \in[0, L], \\
& w(x, 0)=0, \quad w_{t}(x, 0)=0, \quad x \in[0, L], \\
& v(0, t)=p_{0}(t), \quad v(L, t)=p_{L}(t), \quad t \in(0, T), \\
& w(0, t)=0, \quad w(L, t)=0, \quad t \in(0, T), \\
& w_{0}(0, t)=q_{0}(t)-v_{x}(0, t), \quad t \in(0, T), \quad T>0 .
\end{aligned}
$$

Numerically, we next deal with the solutions (5)-(10). Firstly, the FDM is applied on the first part of equation (5) with the initial condition (6) and boundary condition (8), which is similar to the equations in [3] and thus not rewritten here. Then, in the form of $v_{x}(0, t)$, we have

$$
-\frac{\partial v}{\partial x}\left(0, t_{j}\right)=-\frac{4 v_{1, j}-v_{2, j}-3 v_{0, j}}{2 \Delta x}, \quad j=\overline{1, N} \text {. }
$$

A similar way is used for the second part for equations (5), (7), and (9). This step is the main aim of this study:

$$
\begin{aligned}
& w_{i, j+1}=r^{2} w_{i+1, j}+2\left(1-r^{2}\right) w_{i, j}+r^{2} w_{i-1, j}-w_{i, j-1}, \quad i=\overline{1,(M-1)}, j=\overline{1,(N-1)}, \\
& w_{i, 1}=\frac{1}{2} r^{2} w_{i+1,0}+\left(1-r^{2}\right) w_{i, 0}+\frac{1}{2} r^{2} w_{i-1,0}+f_{i} h_{0}, \quad i=\overline{1,(M-1)}, \quad j=0 . \\
& w_{i, 0}=0, \quad i=\overline{0, M}, \quad \frac{w_{i, 1}-w_{i,-1}}{2 \Delta t}=w_{t i, 0}\left(x_{i}\right)=0, \quad i=\overline{1,(M-1)}, \\
& w\left(0, t_{j}\right)=w_{0, j}=0, \quad w\left(L, t_{j}\right)=w_{m, j}=0, \quad j=\overline{0, N},
\end{aligned}
$$

With the additional boundary condition (4) and equation (11) substituted in (10), we get

$$
\frac{\partial w}{\partial x}\left(0, t_{j}\right)=q_{0}\left(t_{j}\right)-\frac{\partial v}{\partial x}\left(0, t_{j}\right), \quad j=\overline{1, N} .
$$

where $\left(v_{i, j} ; w_{i, j}\right):=\left(v\left(x_{i}, t_{j}\right) ; w\left(x_{i}, t_{j}\right)\right)$, such that $x_{i}=i \Delta x(\Delta x=L / M), t_{j}=j \Delta t(\Delta t=T / N)$, for $i=\overline{0, M}, j=\overline{0, N}$ and $r=c \Delta t / \Delta x[2-4,11]$.

Linear system algebra was reached from (12)-(16), i.e $A(w ; f)=b$, when can find $(w(x, t), f(x))$. Moreover, this problem is ill-posed for existences and uniqueness, as proved by [6,9]. For stability, we proposed in this research adding the noise in (4) $q_{0}^{\epsilon}\left(t_{n}\right)=q_{0}\left(t_{n}\right)+\epsilon, n=\overline{1, N}$, then $w_{x}^{\epsilon}\left(t_{n}\right)=$ $q_{0}^{\epsilon}\left(t_{n}\right)-v_{x}\left(0, t_{n}\right)=w_{x}\left(t_{n}\right)+\epsilon$, where $\epsilon$ is a Gaussian normal distribution. The mean of zero was applied, while the standard deviation of $\sigma$ was given by $\sigma=p \% \times \max _{t \in[0, T]}\left|q_{0}(t)\right|[1-4,11]$, and $p \%$ represents the percentage of noise.

Zeroth-order Tikhonov regularization $\underline{b}_{\lambda}=\left(A^{\operatorname{tr}} A+\lambda I\right)^{-1} A^{\operatorname{tr}} \underline{(w ; f)^{\epsilon}}$ was applied after the oscillation being in $(w ; f)$.

\section{Numerical Results and Discussion}

The objective of this study is to test how accurate is $f(x)$, where $w(x, t)$ is the second part of (5), based on the FDM. Let $h(t)=L=T=1$ for simplicity. As mathematicians need to have good background and information of the non-homogenous wave equation, we apply the same example used 
in [3]. In addition, we compare the FDM and separation variables.

$$
\begin{gathered}
u(x, t)=\sin (\pi x)+t+\frac{t^{2}}{2}, \quad f(x)=1+\pi^{2} \sin (\pi x), \quad x \in[0,1], \\
u(x, 0)=u_{0}(x)=\sin (\pi x), \quad u_{t}(x, 0)=v_{0}(x)=1, \quad x \in[0,1], \\
u(0, t)=p_{0}(t)=t+\frac{t^{2}}{2}, \quad u(1, t)=p_{L}(t)=t+\frac{t^{2}}{2}, \quad t \in(0,1] .
\end{gathered}
$$

Over-determination occurs in the left end, where

$$
u_{x}(0, t)=q_{0}(t)=\pi, \quad t \in[0,1] \text {. }
$$

Table 1 and Figure 1 show that the matrix $A$ is an ill-condition, as compared with Table 1 and Figure 2 used in [1], which are quite different because the matrix in [1] comes from separation variables, while in the present work it comes from FDM. Because of having the same values of vx $(0, t)(i . e . ~(11))$ that appeared in [3], the figure is not represented in this research.

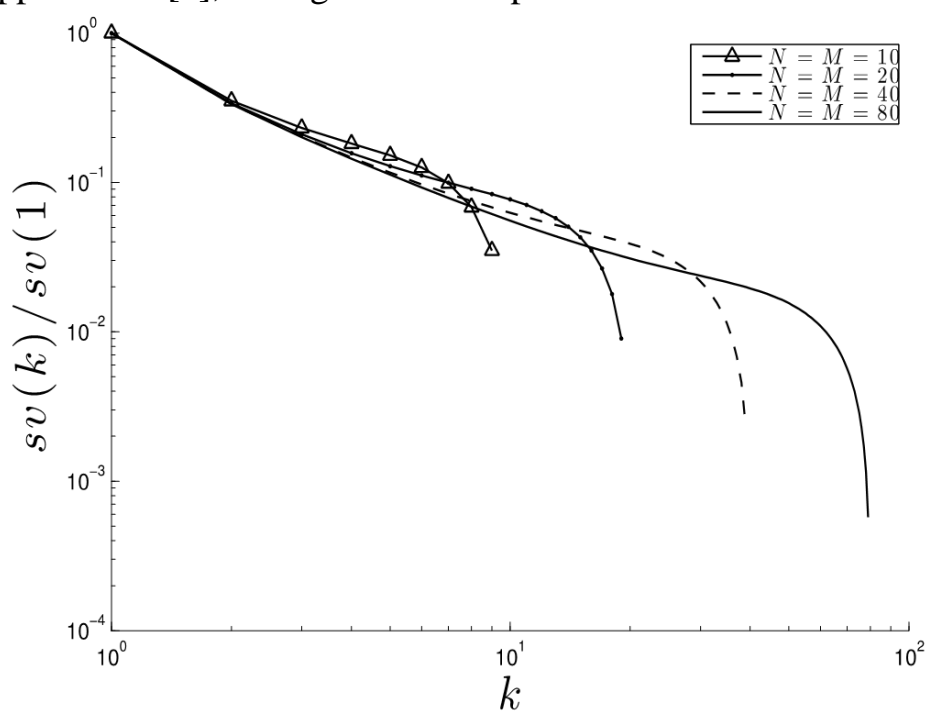

Figure 1- Normalized singular values at $k=1: M-1$ for using FDM (12)-(16) with $\mathrm{M}=\mathrm{N} \in$ $\{20,40,80\}$.

Table 1-Condition number of matrix $A$ in $A(w ; f)=b$

\begin{tabular}{|l|l|l|l|}
\hline$N=M=10$ & $N=M=20$ & $N=M=40$ & $N=M=80$ \\
\hline 28.55125372 & 110.9876489 & 437.9345376 & 1740.258993 \\
\hline
\end{tabular}

Furthermore, from $A(w ; f)=b$, we can obtain the numerical solution $f(x)$. It is clear from Figure 2 that the result gradually converges to the exact one as the value of $N=M \in\{10,20,40,80\}$ increases.

(a)

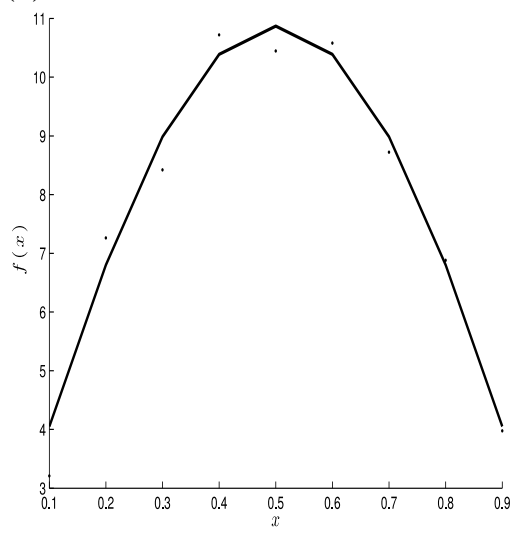

(c) (b)

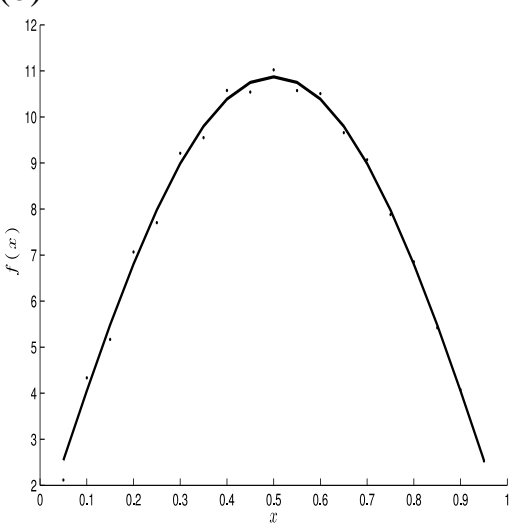

(d) 

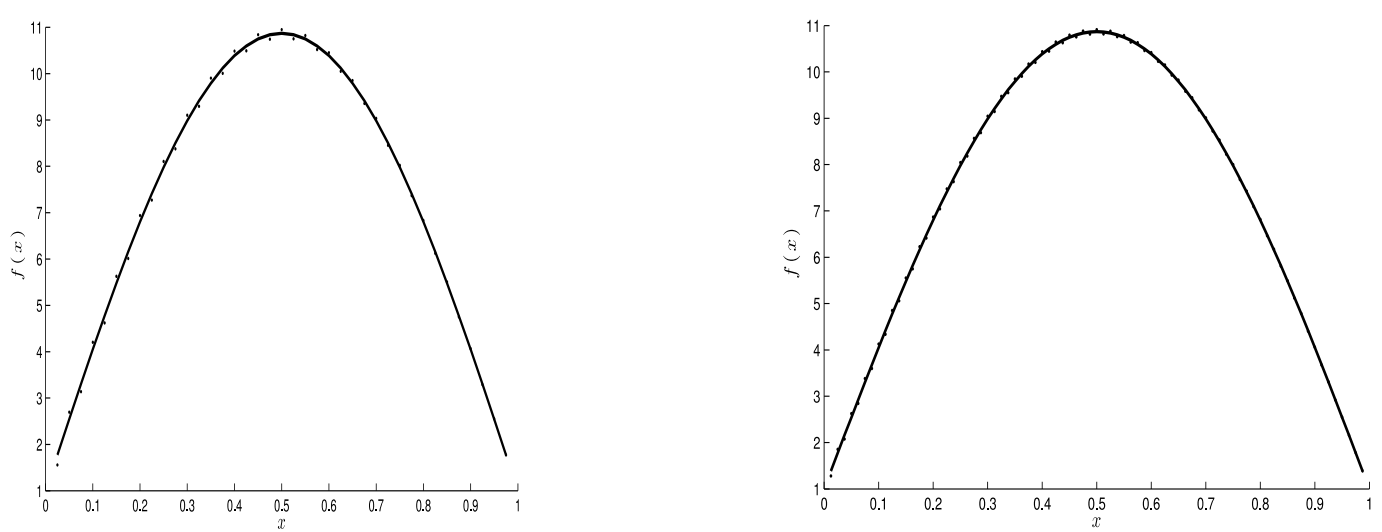

Figure 2-The exact solution (-) as compared to the numerical solution (...), which comes from (12)(16).

As it is popular in science, the inverse force problem is ill-posed. The theorems presented in [6] prove that the solution exists and is unique, only when the third condition remains 'stable', which is proposed in this work. From figure 3, it can be seen that high oscillation acts even when the amount of noise increases, $p \% \in\{1,3,5\} \%$, and add to $w_{x}^{\epsilon}\left(t_{n}\right)=q_{0}^{\epsilon}\left(t_{n}\right)-v_{x}\left(0, t_{n}\right)=w_{x}\left(t_{n}\right)+\epsilon$. It is clear that the solution of $f(x)$ is not well-posed.

The final algorithm used the Tikhonov regularization in the zeroth-order $\underline{b}_{\lambda}=\left(A^{t r} A+\right.$ $\lambda I)^{-1} A^{\operatorname{tr}} \underline{(w ; f)^{\epsilon}}$ to reduce vibration, where $\lambda$ represents the regularization parameter. The min norm error between the exact and numerical noise has placed ||$f_{\text {numerical }}-f_{\text {exact }}||$ as a function of $\lambda$ (see [1$4,11])$ to determine which parameters provided the closest numerical solution. Figure 4 shows that $\lambda=10^{-7}$ reaches the most stable solution for $f(x)$. In addition, different shapes and $\lambda$ values are obtained in the separation variables [3]. Table 2 shows that same $\min \lambda$ has got for $\mathrm{p} \%=\{1,3,5\} \%$ with different values.

(a)

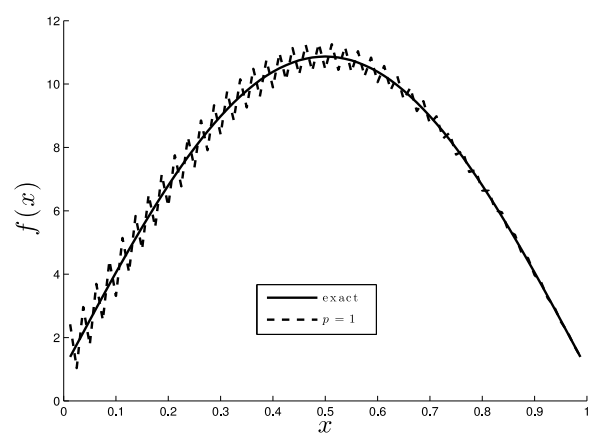

(b)

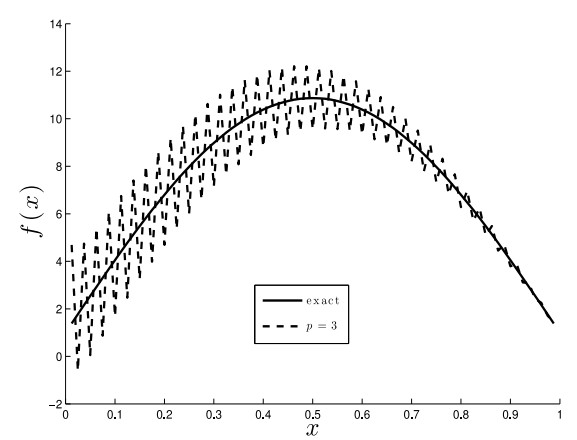

(c)

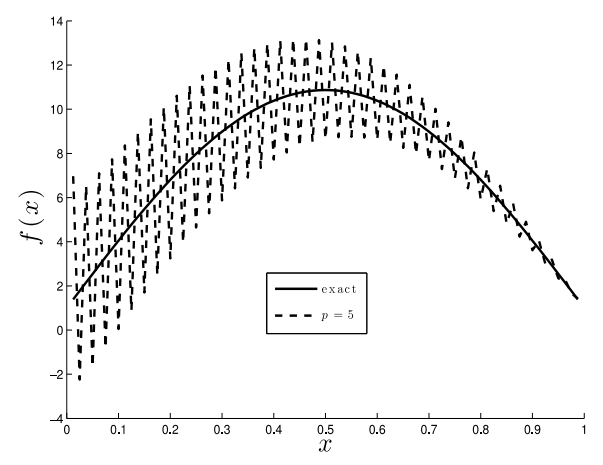

Figure 3- The exact solution for $f(x)(-)$ as compared to the numerical solution (...) with fixed $N=M=80$, which comes from (12)-(15), with $w_{x}^{\epsilon}\left(t_{n}\right)=q_{0}^{\epsilon}\left(t_{n}\right)-v_{x}\left(0, t_{n}\right)=w_{x}\left(t_{n}\right)+\epsilon$ and $p \% \in\{1,3,5\} \%$ 


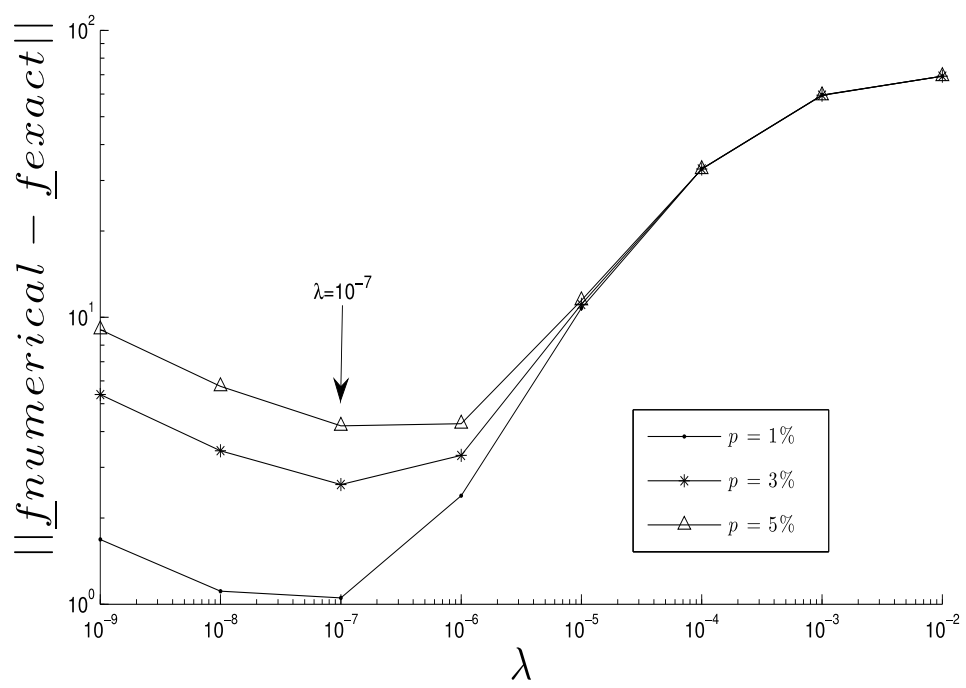

Figure 4-Error $\left\|\mathbf{f}_{\text {numerical }}-\mathbf{f}_{\text {exact }}\right\|$, as a function of $\boldsymbol{\lambda}$, for $\mathbf{N}=\mathbf{M}=\mathbf{8 0}$ and $\mathbf{p} \% \in\{\mathbf{1}, \mathbf{3}, \mathbf{5}\} \%$ noise.

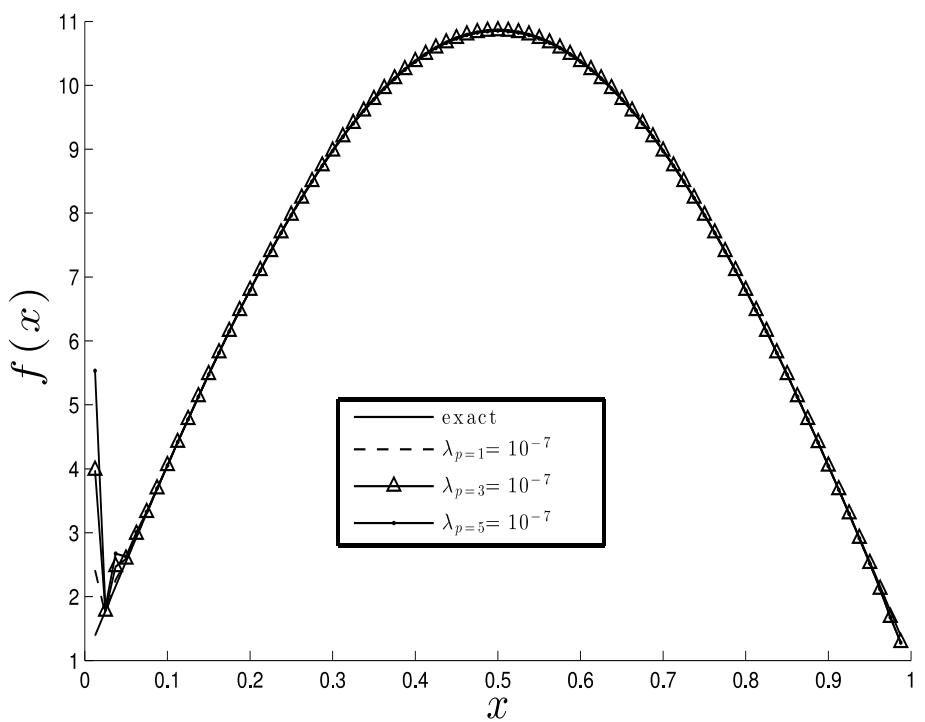

Figure 5- The exact solution for $f(x)$ in comparison with the numerical solution, for $\mathbf{p} \% \in$ $\{\mathbf{1}, \mathbf{3}, \mathbf{5}\} \%$ noise and regularization parameter $\lambda=10^{-7}$.

Table 2- Regularization parameter $\lambda=10^{-7}$ as $\mathbf{p} \%=\{\mathbf{1}, \mathbf{3}, \mathbf{5}\} \%$

\begin{tabular}{|c|c|c|}
\hline$p \%=1 \%$ & $p \%=3 \%$ & $p \%=5 \%$ \\
\hline 1.051405741 & 2.613571308 & 4.185835838 \\
\hline
\end{tabular}

\section{Conclusions}

In this study, we investigated the stable numerical solution for $f(x)$ after considering the finite difference method for both homogeneous and non-homogeneous parts of the wave equation. Additional boundary conditions were supposed along with the initial boundary conditions. Moreover, to obtain the regularization solution, where noise was added to the additional conditions, the zeroth order Tikhonov was tested. Meanwhile, the nearest parameters were examined by min norm errors between the exact and numerical solutions. 


\section{References}

1. Hussein, S. O., \& Lesnic, D. 2014. Determination of a space-dependent source function in the onedimensional wave equation. Electronic Journal of Boundary Elements, 12: 1-26.

2. Hussein, S. O. 2016, January 1. Inverse force problems for the wave equation. Leeds, University Of Leeds, UK. "PhD Thesis"

3. Hussein, S. O. 2020. Splitting the one-dimensional wave equation. Part I: Solving by finitedifference method and separation variables. Baghdad Science Journal, 17: 2078-8665.

4. Hussein, S. O., and Hussein, M.S. 2021. Splitting the one-dimensional wave equation. Part II: Additional data are given by an end displacement measurement. Iraqi Journal of Science, $\mathbf{6 2}$.

5. Tekin, I. 2019. Determination of a time-dependent coefficient in a wave equation with unusual boundary condition. Published by Faculty of Sciences and Mathematics University of Nis Serbia; http://www.pmf.ni.ac.rs/filomat

6. Cannon, J. R., \& Dunninger, D. R. 1970. Determination of an unknown forcing function in a hyperbolic equation from overspecified data. Annali di Matematica Pura ed Applicata, 1: 49-62.

7. Mardanov, M.J., Guliyev, H.F. and Safarova, Z.R. 2020. The problem of starting control with two intermediate moments of observation in the boundary value problem for the hyperbolic equation. Optim Contral Appl Math, 1-10.

8. Yang, F., Zhang, Y., Liu, X. and Li, X. 2020. The quasi-boundary value method for identifying the initial value of the space-time fractional diffusion equation. Act Mathematica Scientia, 40B: 641658.

9. Huanga, C.-H. 2001. An inverse non-linear force vibration problem of estimating the external forces in a damped system with time-dependent system parameters. J Sound Vib, 242: 749-765.

10. Morse, P. M., \& Feshbach, H. 1953. Methods of Theoretical Physics. New York: McGraw-Hill.

11. Hussein, S. O., \& Lesnic, D. 2016. Determination of forcing functions in the wave equation. Part I: the space-dependent case. Journal of Engineering Mathematics, 96: 115-133.

12. Adil, Z., \& Hussein, M.S. 2020. Numerical solution for two-sided Stefan problem. Iraqi Journal of Science, 61(2): 444-452. 